\title{
Shearlet Network-based Sparse Coding Augmented by Facial Texture Features for Face Recognition
}

\author{
Mohamed Anouar Borgi ${ }^{1}$, Demetrio Labate ${ }^{2}$, Maher El'Arbi $^{1}$, \\ and Chokri Ben Amar ${ }^{1}$ \\ 1 REGIM: REsearch Groups on Intelligent Machines, \\ University of Sfax, ENIS, BP 1173 Sfax 3038 Tunisia \\ 2 Department of Mathematics, University of Houston, Houston, TX 77204, USA \\ anoir.borgi@ieee.org, dlabate@math.uh.edu, \\ maher.elarbi@gmail.com, chokri.benamar@ieee.org
}

\begin{abstract}
One open challenge in face recognition (FR) is the single training sample per subject. This paper addresses this problem through a novel approach that combine Shearlet Networks (SN) and PCA called (SNPCA). Shearlet Network takes advantage of the sparse representation (SR) properties of shearlets in biometric applications, Especially, for face coding and recognition. The main contributions of this paper are (1) the combination of the multi-scale representation which capture geometric information to derive a very efficient representation of facial templates, and the use of a PCA-based approach and (2) the design of a fusion step by a refined model of belief function based on the DempsterShafer rule in the context of confusion matrices. This last step is helpful to improve the processing of facial texture features. We compared our algorithm (SNPCA) against SN, a wavelet network (WN) implementation and other standard algorithms. Our tests, run on several face databases including FRGC, Extended Yale B database and others, shows that this approach yields a very competitive performance compared to wavelet networks (WN), standard shearlet and PCA-based methods.
\end{abstract}

Keywords: Shearlet, Shearlets Network, Sparse Coding, Face Recognition.

\section{Introduction}

The current trend in face recognition (FR) emphasizes biometrics which can be collected on the move, so that there is significant interest in more sophisticated and robust methods to go beyond current state of the art FR methods. One of the most successful approaches to template-based face representation and recognition is based on Principal Component Analysis (PCA). However, PCA approximates texture only, while the geometrical information of the face is not properly captured. In addition to PCA, many other linear projection methods have been considered in face recognition applications. The LDA (Linear Discriminant Analysis) has been proposed in [6] as an alternative to PCA. This method

A. Petrosino (Ed.): ICIAP 2013, Part II, LNCS 8157, pp. 611-620, 2013.

(C) Springer-Verlag Berlin Heidelberg 2013 
provides discrimination among the classes, while the PCA deals with the input data in their entirety without paying much attention for the underlying structure. Moreover, to deal with the challenges in practical FR system, active shape model and active appearance model [1] were developed for face alignment; LBP [2] and its variants were used to deal with illumination changes; and Eigenimages [3] 4] and probabilistic local approach [5] were proposed for FR with occlusion.

Recently, FR via sparse representation based classification (SRC) [7] 8] has received more attention as a powerful tool for statistical modeling, sparse representation (or sparse coding) and has been successfully applied to face processing applications. Although SRC performs well when the set of training images is sufficiently large, it is still inadequate for many real world applications where only a single sample per sub-ject is available.

On the other hand, a new generation of multi-scale methods has emerged in recent years which go far beyond traditional wavelets and have been shown to have the potential to provide better performing algorithms in a variety of biometric-based approach. The shearlet system is one notable example of these new classes of multi-scale systems, which has the ability to capture anisotropic information very efficiently, outperforming traditional wavelets. One particularly appealing feature of shearlets is that they combine a multi-scale framework which is particularly effective to capture the geometry of a face, together with a simple mathematical construction which can be associated to a multi-resolution analysis and enables fast numerical processing. We recall that some recent work using shearlets for FR has recently appeared in [9] 10.

In this work, we present a new method for FR, called shearlet network (SN), which is refinement of the classical wavelet network (WN). In this approach, faces will be approximated by a linear combination of weighted sum of shearlets and the weights will be used in the on-line recognition stage to calculate the similarity score between Gallery and probe face. We use PCA-based approach for a fusion step with SN to provide more depth to the facial texture appearance of the face; this fusion is achieved via a model of belief function which will be explained below.

The rest of this paper is organized as follows. In Sec. 2, we briefly describe some background on shearlets. Sec. 3 presents the proposed face coding and recognition method. In Sec. 4, the experimental results of the proposed algorithm are demonstrated and compared with other algorithms. Finally, Sec. 5 concludes this paper.

\section{Background on Shearlets}

The shearlet representation has emerged in recent years as one of the most effective frameworks for the analysis and processing of multidimensional data [1]. The shearlet approach is derived from the theory of wavelets with composite dilatation, a method providing a general framework for the construction of waveforms defined not only at various scales and locations, as traditional wavelets, but also at various orientations and with different scaling factors in each coordinate. As shown in several publications [12] 13] shearlets are 
particularly effective in a number of image processing application, such as denoising and feature extraction, where it is important to capture the geometric information efficiently. As a generalization of the traditional wavelet approach, the continuous shearlet transform [15] is defined as the mapping:

$$
S H_{\psi}(\alpha, s, t)=\left\langle f, \Psi_{\alpha, s, t}\right\rangle, \alpha>0, s \in R, t \in R^{2}
$$

where $\Psi_{\alpha, s, t}(x)=\left|\operatorname{det} M_{\alpha, s}\right|^{-\frac{1}{2}} \Psi\left(M_{\alpha, s}^{-1}(x-t)\right)$ and $M_{\alpha, s}=\left(\begin{array}{cc}\alpha & s \\ 0 & \sqrt{\alpha}\end{array}\right)$

The shearlet transform is a function of three variables : the scale $\alpha$, the shear $\mathrm{s}$ and the translation t. By choosing the generator function appropriately, one can construct a collection of functions $\Psi_{\alpha, s, t}(x)$, called shearlets, which are welllocalized wave-forms at various scales, orientations and locations. One of the main properties of the Continuous Shearlet Transform is its ability to describe very precisely the geometry of the singularities of a 2-dimensional function $\mathrm{f}$. By sampling the Continuous Shearlet Transform $S H_{\psi}(\alpha, s, t)$ on an appropriate discrete set we obtain a discrete transform. Specifically, $M_{a l p h a, s}$ is "discretized" as $M_{j, l}=B_{l} A^{j}$, where $\mathrm{B}=\left(\begin{array}{ll}1 & 1 \\ 0 & 1\end{array}\right), \mathrm{A}=\left(\begin{array}{ll}4 & 0 \\ 0 & 2\end{array}\right)$ are the shear matrix and the anisotropic dilation matrix, respectively. Hence, the discrete shearlets are the functions of the form:

$$
\psi j, l, k(x)=2^{\frac{3 j}{2}} \psi\left(B_{l} A^{j} x-k\right), j \geq 0,-2^{j} \leq l \leq 2^{j}-1, k \in Z^{2}
$$

Figure1illustrates the two-level shearlet decomposition of a face image from Yale database, where the first-level and and the second-level decomposition generates 8 sub-bands corresponding to the different directional bands.

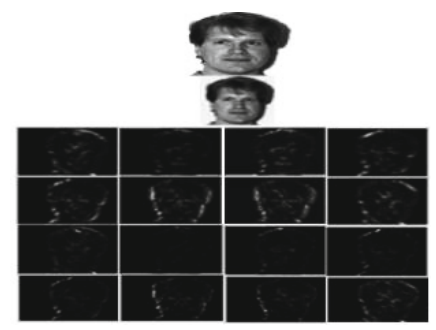

Fig. 1. The top image is the original Face image. The image below the top image contains the approximation shearlet coefficients. Images of the detail shearlet coefficients are shown below.

\section{The Proposed Approach}

The pipeline of all these stages is illustrated in Figure 2 . 


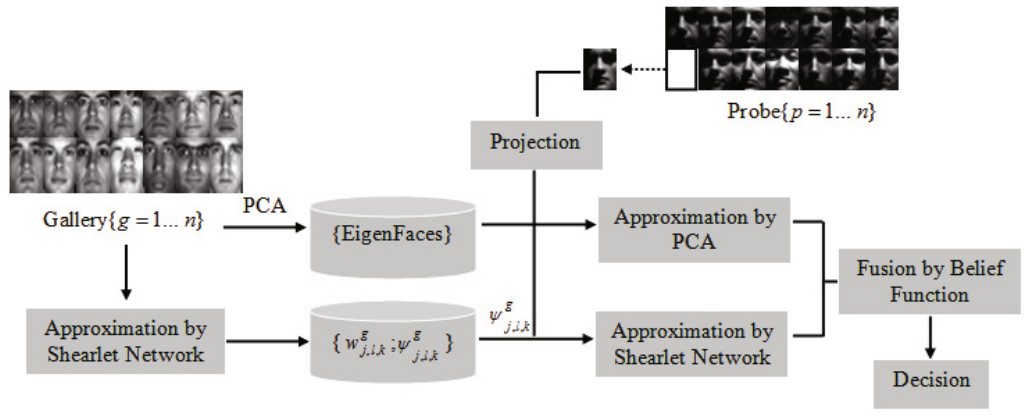

Fig. 2. Framework of 2D face recognition by Shearlet Networks and PCA

The Gallery faces are approximated by a shearlet network (SN) to produce a compact biometric signature constituted by shearlets and their weights, which will be used to match a Probe with all faces in the Gallery. In the recognition stage, the Test (Probe) face is projected on the shearlet network (SN) of the Gallery face and new weights specific to this face are produced. The family of shearlets remains then unchanged (that of the Gallery face). Finally, a similarity score is produced by comparing the weights of the Gallery face with the weights of the test face. In another section of the pipeline, PCA is used for FR [28] in order to generate the eigenfaces from Gallery database which will be operated for the projection step of Probe database. Those two matchers, SN and PCA, will generate two confusion matrices which are used to fill two belief mass matrices with belief functions. Finally, we combined those matrices using a conjunction fusion rule. Detailed description of the above step is given in next subsections.

\subsection{Approximation by Shearlet Network (SN)}

A SN is a combination of the RBF neural network and the shearlet decomposition. The SN algorithm approximates a 2D face image $\mathrm{f}$ using a linear combination of shearlet functions in the network that are multiplied by corresponding weights according to:

$$
\breve{f}=\sum_{j, l, k} w_{j, l, k} * \psi_{j, l, k}
$$

where $\mathrm{f}$ is the face image, $\breve{f}$ is the face image approximation, $w_{j, l, k}$ are the weights and $\psi_{j, l, k}$ are the shearlet functions. An important part in the design of this method is the weights optimization. For an image f, an energy (error) function is specified which is minimized through a learning process with respect to the desired SN parameters.

$$
E=\min _{i}\left\|f_{i}-\sum_{j, l, k} w_{j, l, k} * \psi_{j, l, k}\right\|
$$

The mother shearlet used in our work to construct the family $\psi_{j, l, k}$ is the second derivative of the beta function [16]. The algorithm used for the FR by SN is based 
on the dyadic sampling necessary for building the shearlet candidates library to use in the network and the frames theory for the weights calculations [17. By sampling the continuous shearlet transform $S H_{\psi}(\alpha, s, t)$ on an appropriate discretization lattice for the scaling, shear, and translation parameters $(\alpha, s, t)$, one obtains a discrete transform which is associated to a Parseval (tight) frame for $L^{2}\left(R^{2}\right)$. Indeed, we obtain a discrete system of shearlets $\psi_{j, l, k}$, for $j, l \in$ $Z, k \in Z^{2}$ which, for an appropriate generator [11], satisfies the reproducing formula:

$$
f=\sum_{j, l \in Z, k \in Z^{2}}\left\langle f, \psi_{j, l, k}\right\rangle \psi_{j, l, k}
$$

In the optimization stage, a shearlet coefficient from the library is processed through the hidden layer of the network and used to update the weights. The low frequency shearlets associated with the coarse approximation level of the image to be analyzed are processed first; the high frequency shearlets are used next to refine the approximated image. In order to know if a shearlet (n) will be an activation function of a new "neuron", we must verify if it is a linear combination of the other (n-1) shearlets of the network. In other words, it must be linearly independent with respect to the other (n-1) shearlets. The calculation of the weights connection in every stage is obtained by projecting the image to be analyzed on a family of shearlets. Note however that the beta shearlet (shearlets whose generator is a beta function) are not necessarily orthogonal. Hence, for a given family of shearlets it is not possible to calculate the weights by direct projection of the function. At every stage of the optimization process we need to know the dual family of the shearlets forming our shearlet network. The dual shearlet family is calculated by the formula:

$$
\breve{\psi}_{j, l, k}^{i}=\sum_{m=1}^{N}\left(\Psi_{i, m}\right)^{-1}\left(\psi_{j, l, k}\right)^{m} \text { with } \Psi_{i, m}=\left\langle\psi_{j, l, k}^{i}, \psi_{j, l, k}^{m}\right\rangle
$$

It is clear that the quality of reconstructed image increases with the number of shear-lets used as illustrated in Figure 3 .

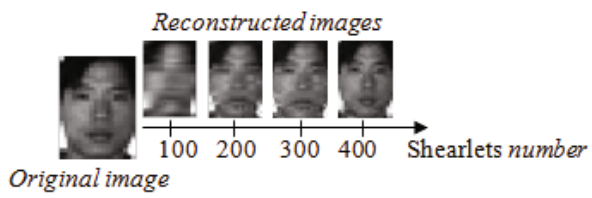

Fig. 3. Example of a $2 \mathrm{D}$ face and the corresponding reconstructed images by beta shearlet networks

\subsection{Fusion by Belief Function}

A step to normalize similarity matrices is essential, before combination, to generate meaningful distances from the two matchers: SN and PCA. In this paper, we use the Min-Max normalization, which has the following formula: 


$$
d^{\prime}=\frac{d-d_{\min }}{d_{\max }-d_{\min }}
$$

where $d_{\max }, d_{\min }, \mathrm{d}$ and d' denote respectively the maximum, the minimum, the original and the normalized distances. In the second step, we use a Bayesian assignment [18] to compute the belief mass functions values from the confusion matrix. Finally, a conjunctive combination rule [19], defined by the following equation is used to fuse confusion matrices.

$$
\forall A \subseteq \Omega, m_{S N \cap P C A}(A)=m_{S N} \cap m_{P C A}(A)=\sum_{B \cap C=A} m_{S N}(B) m_{P C A}(C)
$$

where $m_{S N}$ and $m_{P C A}$ denote the two Belief mass functions associated to SN and PCA.

\section{Experimental Results}

We run our experiments using standard benchmark face databases to evaluate the performance of the proposed approach. We used the Extended Cohn-Kanade $(\mathrm{CK}+)$ [20, Georgia Tech [21, FEI 22], Extended YALE B [23] and FRGC v1 24] face databases. All the images are copped except the Extended CohnKanade $(\mathrm{CK}+)$ database and resized to 2732. In this paper, we chose to select randomly the face image both for Gallery and Probe database. A description of the databases used in Table 1 and examples of subjects (Gallery and Probe) in Figure 4 .

Table 1. Description of the different databases used

\begin{tabular}{|c|c|c|c|c|c|}
\hline Database & CK+ & Georgia Tech & FEI & Yale B & FRGC v1 \\
\hline Database Size & 593 & 750 & 2800 & 16128 & 4003 \\
\hline Subject number & 123 & 50 & 200 & 38 & 152 \\
\hline
\end{tabular}

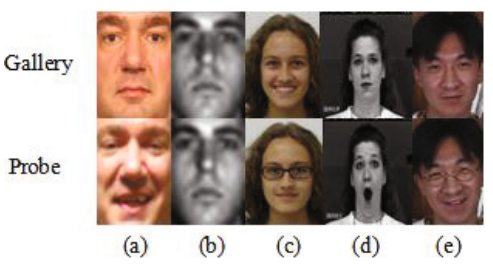

Fig. 4. A subject from Gallery and Probe with different databases. (a) FRGC. (b) Yale B. (c) FEI. (d) Extended CK+. (e) Georgia Tech. 
Most work in the literature uses multiple training samples per subject. However, an emerging tendency in FR is to use Single Training Sample per Subject (STSS) [25] 26] 27, which is a more challenging problem. By applying such strategy to the images from the databases indicated above, we obtain a similarity matrix of 38x38 comparisons for Extended YALE B, 123x123 comparisons for Extended Cohn-Kanade (CK+), 152x152 comparisons for FRGCv1, 200x200 comparisons for FEI and 50x50 comparisons for Georgia Tech, which significantly reduce the computational complexity of the algorithm compared to traditional multiple training samples per subject. Hence, the similarity values located in the diagonal of the matrix are intra-class (the same person) and the others are inter-class (different persons) or imposter access. In this paper, we considered the problems of face coding, recognition and authentication. Results are provided in next subsections.

\subsection{Face Coding Evaluation}

We use some standard quality measurement tool, such as the Peak Signal to Noise Ratio (PSNR) and the Normalized Cross Correlation (NCC). We compared the two approaches, e.g, SN vs. WN, and to the standard shearlet representation. We test with 3 face images for the same person from YALE database. The SN (table 2) is found to outperform the two other methods that we considered.

Table 2. PSNR and NCC of different methods. (higher is better)

\begin{tabular}{|c|c|c|c|c|c|c|}
\hline Method & PSNR & NCC & PSNR & NCC & PSNR & NCC \\
\hline Shearlet & 27.1227 & 0.9913 & 26.1698 & 0.9853 & 26.1582 & 0.9861 \\
\hline WN & 36.9600 & 0.9967 & 33.4986 & 0.9942 & 32.5192 & 0.9931 \\
\hline SN & 37.7788 & 0.9973 & 36.2613 & 0.9970 & 35.8314 & 0.9962 \\
\hline
\end{tabular}

In order to have the same experimental protocol and conditions for FC we chose a shearlets number (SN) equal to wavelets number (WN) equal to filters number of shearlet-based approach. We have also plotted the reconstruction error versus the number of employed wavelets (WN) and shearlets of (SN). Details for these results are not reported here for reasons of space.

\subsection{Face Recognition and Authentication Using STSS}

The following experiments illustrate our results regarding authentication and recognition tasks. In these experiments, similarity matrices and mass belief functions matrices are produced, rank-one recognition rates (Rank-1) are computed and evaluation Receiver Operator Characteristic (ROC) curves are plotted. Table 3 summarize the rank-one recognition rates (Rank-1). SNPCA, the proposed approach, presents a Rank-1 which outperforms SN, WN, PCA approaches with whole the face databases. 
Table 3. Rank-1 with different subsets database (\%)

\begin{tabular}{|c|c|c|c|c|c|}
\hline Method & Extended CK+ & FEI & Georgia Tech & Yale B & FRGC v1 \\
\hline PCA & 80.49 & 59.00 & 6.00 & 51.50 & 12.50 \\
\hline WN & 91.87 & 78.50 & 16.00 & 86.84 & 23.03 \\
\hline SN & 95.94 & 79.50 & 26.00 & 97.37 & 34.87 \\
\hline SNPCA & 96.75 & 85.50 & 30.00 & 97.37 & 43.50 \\
\hline
\end{tabular}

The authentication experiments are illustrated in Figure 5, one image for each one of the 5 face database considered. Each figure shows an ROC curve, where the $\mathrm{x}$-axis represents the False Accept rate. The figures shows that SNPCA provides consistently the best performance.

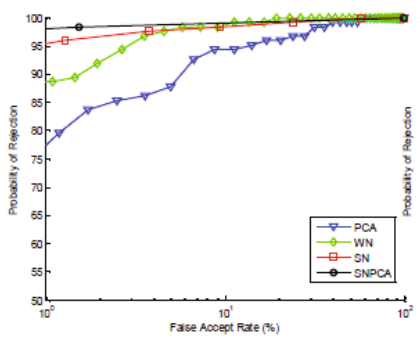

(a)

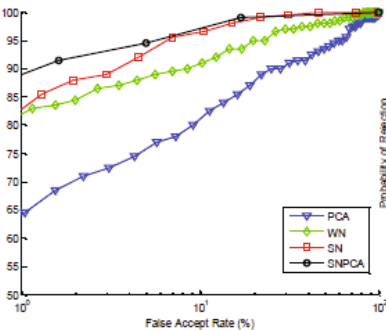

(b)

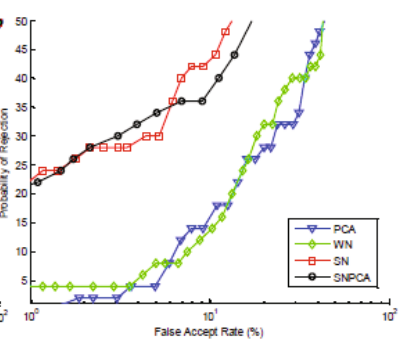

(c)

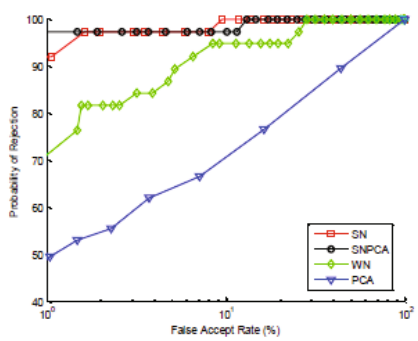

(d)

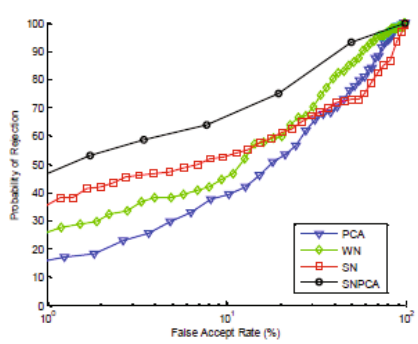

(e)

Fig. 5. ROC curves with (a) Extended Cohn-Kanade (CK+) database, (b) FEI database, (c) Georgia Tech database , (d) Extended Yale B database, (e) FRGC v1 database

From Table 2, we notice that SN gives better results in terms of PSNR and NC as compared to WN and shearlet coding. This is due to the superior ability of SN to capture the geometry of the data. Also for FR applications the SN performs better than PCA and WN as shown by Table 3 and Figure 5 . Moreover, the combination of PCA and SN (SNPCA) using belief functions improves considerably the recognition rates. This shows that features (texture feature for PCA and anisotropic feature for SN) from matchers based on different approaches can be effectively combined. 


\section{Conclusion}

This paper presents a novel sparse coding (SC) model for robust face recognition (FR) called Shearlet Network (SN). This method was performed via fusion step by PCA-based approach to provide more depth to facial texture feature, using a refined model of belief function based on the Dempster-Shafer rule. One important advantage of SNPCA is its robustness to various types of challenging scenarios of FR (pose, expression and illumination). This paper focused on the challenging problem of a single training sample per subject. In order to illustrate and validate our approach we used the Extended Cohn-Kanade (CK+), Georgia Tech, FEI, Extended YALE B and FRGC v1 face databases. Our results show that our approach is very competitive in terms of recognition rate with respect to other standard and state-of-the-art methods.

Acknowledgments. The authors (1) would like to acknowledge the financial support of this work by grants from General Direction of Scientific Research (DGRST), Tunisia, under the ARUB program. D.L.(2) acknowledges partial support by NSF DMS 1005799 and DMS 1008900.

\section{References}

1. Lanitis, A., Taylor, C.J., Cootes, T.F.: Automatic Interpretation and Coding of Face Images Using Flexible Models. IEEE Trans. Pattern Analysis and Machine Intelligence 19(7), 743-756 (1997)

2. Ahonen, T., Hadid, A., Pietikainen, M.: Face description with local binary patterns: Application to face recognition. IEEE Trans. Pattern Analysis and Machine Intelligence 28(12), 2037-2041 (2006)

3. Leonardis, A., Bischof, H.: Robust recognition using eigenimages. Computer Vision and Image Understanding 78(1), 99-118 (2000)

4. Chen, S., Shan, T., Lovell, B.C.: Robust face recognition in rotated eigenspaces. In: Proc. Intl Conf. Image and Vision Computing, New Zealand (2007)

5. Martinez, A.M.: Recognizing Imprecisely localized, partially occluded, and expression variant faces from a single sample per class. IEEE Trans. Pattern Analysis and Machine Intelligence 24(6), 748-763 (2002)

6. Lu, J., Plataniotis, K.N., Venetsanopoulos, A.: Face recognition using LDA-based algorithms. IEEE Trans. Neural Networks 14(1), 195-200 (2003)

7. Yang, M., Zhang, D.: Jian Yang, Zhang, D.: Robust sparse coding for face recognition. In: Proc. IEEE Conf. Computer Vision and Pattern Recognition (2011)

8. Yang, M., Zhang, L., Yang, J., Zhang, D.: Regularized Robust Coding for Face Recognition. IEEE Trans. on Image Processing (2013)

9. Schwartz, W.R., da Silva, R.D., Davis, L.S., Pedrini, H.: A Novel Feature Descriptor Based on the Shearlet Transform. In: IEEE International Conference on Image Processing, pp. 1033-1036 (2011)

10. Schwartz, W.R., Guo, H., Choi, J., Davis, L.S.: Face Identification Using Large Feature Sets. IEEE Transactions on Image Processing 21(4), 2245-2255 (2012)

11. Negi, P.S., Labate, D.: 3D discrete shearlet transform and video processing. IEEE Trans. Image Process. 21(6), 2944-2954 (2012) 
12. Easley, G.R., Labate, D.: Critically sampled wavelets with composite dilations. IEEE Trans. Image Process. 21(2), 550-561 (2012)

13. Easley, G.R., Labate, D., Patel, V.: Directional multiscale processing of images using wavelets with composite dilations. J. Math. Imag. Vision (to appear, 2013)

14. Yi, S., Labate, D., Easley, G.R., Krim, H.: A Shearlet approach to Edge Analysis and Detection. IEEE Trans. Image Process. 18(5), 929-941 (2009)

15. Kutyniok, G., Labate, D.: Resolution of the wavefront set using continuous shearlets. Trans. Amer. Math. Soc. 361, 2719-2754 (2009)

16. Ben Amar, C., Zaied, M., Alimi, M.A.: Beta Wavelets. Synthesis and application to lossy image compression. Journal of Advances in Engineering Software 36(7), 459-474 (2005)

17. Zaied, M., Jemai, O., Ben Amar, C.: Training of the Beta wavelet networks by the frames theory: Application to face recognition. In: International Workshops on Image Processing Theory, Tools and Applications, Tunisia (November 2008)

18. Mercier, D., Cron, G., Denoeux, T., Masson, M.: Fusion of multi-level decision systems using the Transferable Belief Model. In: Proc. FUSION, Philadelphia, PA, USA (July 2005)

19. Denoeux, T.: Conjunctive and Disjunctive Combination of Belief Functions Induced by Non Distinct Bodies of Evidence. Artificial Intelligence 172, 234-264 (2008)

20. Lucey, P., Cohn, J.F., Kanade, T., Saragih, J., Ambadar, Z., Matthews, I.: The Extended Cohn-Kande Dataset $(\mathrm{CK}+)$ : A complete facial expression dataset for action unit and emotion-specified expression. In: Proc. CVPR, Workshop H CBA (2010)

21. Georgia Tech Face Database, ftp://ftp.ee.gatech.edu/pub/users/hayes/facedb/

22. Thomaz, C.E., Giraldi, G.A.: A new ranking method for Principal Components Analysis and its application to face image analysis. Image and Vision Computing 28(6), 902-913 (2010)

23. Georghiades, A.S., Belhumeur, P.N.: From Few to Many: Illumination Cone Models for Face Recognition under Variable Lighting and Pose. IEEE Trans. on. PAMI 23(6), 643-660 (2001)

24. Phillips, P.J., Flynn, P.J., Scruggs, T., Bowyer, K.W., Chang, J., Hoffman, K., Marques, J., Min, J., Worek, W.: Overview of the face recognition grand challenge. In: Proc. CVPR 2005, vol. 1, pp. 947-954 (June 2005)

25. Yang, M., Zhang, L., Zhang, D.: Efficient Misalignment Robust Representation Representation for Real-Time Face Recognition Classification. In: Fitzgibbon, A., Lazebnik, S., Perona, P., Sato, Y., Schmid, C. (eds.) ECCV 2012, Part I. LNCS, vol. 7572, pp. 850-863. Springer, Heidelberg (2012)

26. Zhu, P., Zhang, L., Hu, Q., Shiu, S.C.K.: Multi-scale Patch based Collaborative Representation for Face Recognition with Margin Distribution Optimization. In: Fitzgibbon, A., Lazebnik, S., Perona, P., Sato, Y., Schmid, C. (eds.) ECCV 2012, Part I. LNCS, vol. 7572, pp. 822-835. Springer, Heidelberg (2012)

27. Su, Y., Shan, S.G., Chen, X.L., Gao, W.: Adaptive Generic Learning for Face Recogni-tion from a Single Sample per Person. In: Proc. CVPR (2010)

28. Mian, A.S., Bennamoun, M., Owens, R.: 2D and 3D Multimodal Hybrid Face Recognition. In: Leonardis, A., Bischof, H., Pinz, A. (eds.) ECCV 2006. LNCS, vol. 3953, pp. 344-355. Springer, Heidelberg (2006) 